\title{
Utilização de marcadores do processo inflamatório no diagnóstico precoce de sepse e sua correlação com o escore de sepse e sobrevivência em potros neonatos provenientes de éguas com placentite
}

Luciana de Araujo Borba ${ }^{[a]}$, Carlos Eduardo Wayne Nogueira[a], Fernanda Maria Pazinato[a], Bruna dos Santos Suñe Moraes ${ }^{[a]}$, Vitória Müller ${ }^{[a]}$, Igor Frederico Canisso ${ }^{[b]}$, Bruna da Rosa Curcio ${ }^{[a]}$

[a] Universidade Federal de Pelotas (UFPel), Pelotas, RS, Brasil
[b] University of Illinois at Urbana-Champaign (UIUC), Urbana, IL, Estados Unidos

*Autor correspondente

e-mail: luaraujo_sm@hotmail.com

\section{Resumo}

A placentite é uma importante causa de aborto e morte neonatal em equinos. 0 desenvolvimento de sepse neonatal é uma consequência comum da placentite, sendo considerada a maior causa de morte em potros até os sete dias de vida. Entretanto, poucos estudos existem acerca do diagnóstico precoce e prognóstico desses potros. O objetivo deste estudo foi avaliar a concentração da proteína amilóide A sérica (SAA), leucócitos totais, fibrinogênio e lactato, e verificar a sua correlação com o escore de sepse e com a sobrevivência em potros nascidos de éguas com placentite ascendente induzida experimentalmente. Tem-se como hipótese que esses marcadores apresentam-se em maiores concentrações no plasma de potros nascidos de éguas com placentite quando na presença de sepse, e que existe correlação com o escore de sepse e com a sobrevivência. Trinta e cinco potros foram utilizados neste estudo. Sete potros saudáveis compuseram o grupo saudável $(n=7)$. Vinte e oito potros nascidos de éguas com placentite foram classificados de acordo com o escore de sepse: não sépticos ( $n=19)$, com escore de sepse menor que 11, e potros sépticos $(n=9)$, com escore de sepse igual ou acima de 11 . Foram realizadas coletas sanguíneas ao nascimento, com 24 e $48 \mathrm{~h}$ de vida para mensuração dos níveis de proteína amiloide A sérica, leucócitos totais, lactato e fibrinogênio. A comparação entre grupos foi realizada através do teste de Kruskal-Wallis, devido à distribuição não paramétrica verificada no teste de Shapiro-Wilk. A comparação da taxa de sobrevivência entre os grupos foi realizada através do Teste Exato de Fisher. A associação entre as variáveis foi avaliada através do teste de correlação de Spearman. Para realização da análise estatística foi utilizado o software SPSS 20.0. A taxa de sobrevivência foi menor nos potros sépticos $(22 \%, \mathrm{n}=2 / 9 ; \mathrm{P}<0.001)$ em relação aos não sépticos (89\%, $n=17 / 19)$ e grupo controle (100\%, n = 7/7). Embora sem diferença entre os grupos, concentrações mais elevadas de proteína amiloide A sérica foram observadas nos animais do grupo séptico em relação aos grupos 
controle e não sépticos. Os valores de leucócitos totais, lactato e fibrinogênio não apresentaram diferença entre os grupos ao nascimento. Nas $24 \mathrm{~h}$ e $48 \mathrm{~h}$, somente as concentrações de lactato foram maiores no grupo séptico $(\mathrm{P}<0,001)$ em relação aos grupos saudáveis e não sépticos. Foi observada correlação entre o escore de sepse e a sobrevivência $(\rho=-0,56 ; \mathrm{P}<0,001)$. Ao nascimento, constatou-se correlação positiva entre a proteína amiloide A sérica e o escore de sepse $(\rho=+0,43 ; \mathrm{P}=0.023)$ e entre os valores de proteína amiloide A sérica e leucócitos totais $(\rho=-0,49 ; \mathrm{P}=0,04)$. Nas 24 e 48 horas, observou-se correlação entre o escore de sepse e as concentrações de lactato $(\rho=0,69$; $\mathrm{P}<0.001$ e $\rho=0,56$; P $<0,001$, respectivamente). Nas 48 horas de vida foi observada, ainda, correlação positiva entre os valores de leucócitos totais e a sobrevivência $(\rho=0.46 ; P=0,01)$. Não foi observada correlação entre as concentrações de SAA e a sobrevivência. Neste estudo observamos que, embora sem diferença entre grupos, os valores de amiloide A sérica foram mais elevados ao nascimento, e que existe correlação positiva entre a SAA e o escore de sepse. As concentrações de fibrinogênio e leucócitos totais não mostraram ser marcadores úteis de sepse nesta população de potros. Os valores de lactato não demonstraram ser um marcador sensível ao nascimento, porém, a partir das 24 horas podem ser utilizados como indicador do processo séptico.

Palavras-chave: Proteína amilóide A sérica. Lactato. Fibrinogênio. 